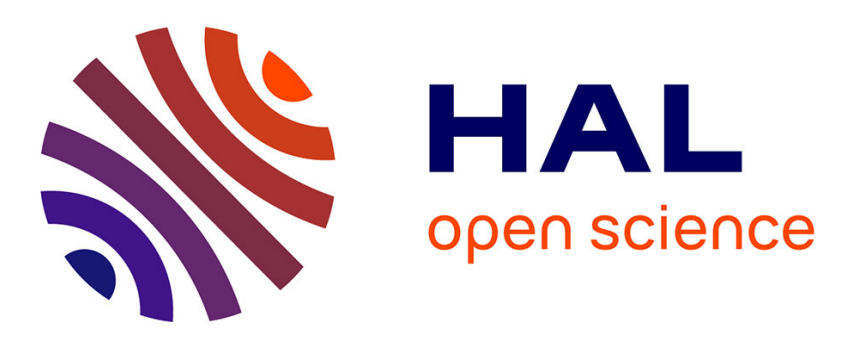

\title{
A mixture approach to the acoustic properties of a macroscopically inhomogeneous porous aluminum in the equivalent fluid approximation
}

Carlos Javier Sacristan Lopez-Mingo, Thomas Dupont, Olivier Sicot, Philippe Leclaire, Kévin Verdière, Raymond Panneton, Xiao-Lu Gong

\section{To cite this version:}

Carlos Javier Sacristan Lopez-Mingo, Thomas Dupont, Olivier Sicot, Philippe Leclaire, Kévin Verdière, et al.. A mixture approach to the acoustic properties of a macroscopically inhomogeneous porous aluminum in the equivalent fluid approximation. Journal of the Acoustical Society of America, 2016, 140 (4), pp.2847-2855. 10.1121/1.4965300 . hal-01396085

\section{HAL Id: hal-01396085 \\ https://hal.science/hal-01396085}

Submitted on 15 Nov 2016

HAL is a multi-disciplinary open access archive for the deposit and dissemination of scientific research documents, whether they are published or not. The documents may come from teaching and research institutions in France or abroad, or from public or private research centers.
L'archive ouverte pluridisciplinaire HAL, est destinée au dépôt et à la diffusion de documents scientifiques de niveau recherche, publiés ou non, émanant des établissements d'enseignement et de recherche français ou étrangers, des laboratoires publics ou privés. 


\title{
A mixture approach to the acoustic properties of a macroscopically inhomogeneous porous aluminum in the equivalent fluid approximation
}

\author{
C. J. Sacristan, T. Dupont, O. Sicot, and P. Leclaire \\ Département de Recherche en Ingénierie des Véhicules pour l'Environnement - EA1859, \\ Université Bourgogne Franche Comté, Nevers F-58000, France \\ K. Verdière and R. Panneton \\ Groupe d'acoustique de l'Université de Sherbrooke, Département de Génie Mécanique, \\ Université de Sherbrooke, Sherbrooke, Quebec J1K 2R1, Canada \\ X. L. Gong \\ Laboratoire des Systèmes Mécaniques et Ingénierie Simultanée, Institut Charles Delaunay, Unité Mixte de \\ Recherche CNRS UMR 6281, Université de Technologie de Troyes, 10010 Troyes, France
}

(Received 6 September 2015; revised 25 September 2016; accepted 6 October 2016; published online 21 October 2016)

\begin{abstract}
The acoustic properties of an air-saturated macroscopically inhomogeneous aluminum foam in the equivalent fluid approximation are studied. A reference sample built by forcing a highly compressible melamine foam with conical shape inside a constant diameter rigid tube is studied first. In this process, a radial compression varying with depth is applied. With the help of an assumption on the compressed pore geometry, properties of the reference sample can be modelled everywhere in the thickness and it is possible to use the classical transfer matrix method as theoretical reference. In the mixture approach, the material is viewed as a mixture of two known materials placed in a patchwork configuration and with proportions of each varying with depth. The properties are derived from the use of a mixing law. For the reference sample, the classical transfer matrix method is used to validate the experimental results. These results are used to validate the mixture approach. The mixture approach is then used to characterize a porous aluminium for which only the properties of the external faces are known. A porosity profile is needed and is obtained from the simulated annealing optimization process.
\end{abstract}

\section{INTRODUCTION}

The variations with depth of the macroscopic parameters of a porous layer can modify significantly its acoustic properties. Rigid frame macroscopically inhomogeneous porous media saturated by air were studied by De Ryck et al. ${ }^{1,2}$ With the help of the state vectors formalism and of Peano series, this problem was extended to the onedimensional (1D) problem (in the thickness) of a macroscopically inhomogeneous poroelastic layer by Gautier et $a .^{3}$ This formalism was also used by Geslain et $a l^{4}$ to describe porous materials with continuously stratified porosity. In the present article, the acoustical properties of a macroscopically inhomogeneous porous material are studied through the use of a static radial compression that can vary in the thickness of a cylindrical sample. The results are then applied to the study of an inhomogeneous aluminium foam.
The acoustical properties of compressed air-saturated porous fibrous material were studied by Castagnède et al. ${ }^{5}$ who modelled a fibrous material as a collection of parallel infinite uncurved fibres undergoing a static 1D uniaxial compression or a $2 \mathrm{D}$ radial compression. In both cases the propagation axis of the incident sound field was perpendicular to the fibres. In the $1 \mathrm{D}$ compression case, the compression is applied along the propagation axis. In the $2 \mathrm{D}$ case, the compression is applied to a homogenization cylinder whose axis is parallel to the fibres. More recently, Wang et al. ${ }^{6}$ provided further development by accounting for the elastic properties of the compressed fibrous material in the $1 \mathrm{D}$ compression configuration. Also in the 1D configuration, Campolina et al. ${ }^{7}$ studied the transmission loss of a metal plate covered by a compressed fibrous material in a diffuse incident sound field and in the rigid or limp frame approximation. In the case of a highly porous polyurethane foams, Geslain et $a l .{ }^{8}$ studied the effect of a 1D compression along the propagation axis on the absorption coefficient.

In a first step in the present work, a reference inhomogeneous material is built by forcing a highly porous tapered cylinder of melamine foam inside a rigid tube (the acoustic impedance tube). In this process, a static 2D compression gradually varying with depth is applied radially to the 
propagation axis, which corresponds to the axis of the cylindrical sample. With the help of an assumption on the pore geometry during the compression, it is possible to propose a model for the physical properties considered anywhere in the thickness. The classical transfer matrix method (TMM) approach (see Allard and Atalla, ${ }^{9}$ Chap. 11) can then be applied to determine theoretically the absorption coefficient and the transmission loss. Experimental results on the transmission loss are compared to theoretical predictions in the rigid or limp frame approximation.

For the aluminium foam, only the physical properties of the external faces are known and the classical TMM approach cannot be used. A new approach is proposed in which the inhomogeneous material is considered to be constituted of a mixture of two materials $A$ and $B$ with volume proportions of each varying through the thickness of the layer as material $A$ is progressively replaced by material $B$. At every position in the thickness of the sample, thin slices of materials $A$ and $B$ of thickness $d x$ are supposed to be placed in parallel relatively to the propagation axis in a patchwork configuration with depth varying surface ratio. The acoustical properties of each slice is then determined by using a mixing rule discussed in Sec. II. In the case of the reference material only, the prediction of the mixture approach coupled with the mixing rule can be confronted to the experimental results and to the predictions of the classical TMM.

The mixing can be carried out using a rule derived from the general theory of homogenization referred to as first order homogenization procedure ${ }^{10,11}$ and provides the effective density and bulk modulus of a patchwork of two porous media from, respectively, the harmonic average of the densities of their constituents and of their bulk moduli. The results presented here are valid at normal incidence. Also, under certain circumstances, it is shown in the Appendix that the classical admittance sum method or ASM provides similar results as the first order homogenization procedure. The ASM method is imbedded in a recently developed transfer matrix method involving elements placed in parallel ( $\mathrm{P}$ TMM) (see Verdière et al. ${ }^{12,13}$ ).

In Sec. II, the reference inhomogeneous material is presented. In Sec. III, the physical parameters are modelled as functions of the volume proportion of each material. The acoustic properties of a compressed tapered cylinder are studied in Sec. IV. A comparison between experimental results with theoretical predictions provided by the classical transfer matrix method is presented. The same experimental results are then compared to the predictions provided by the mixture approach for their validation. The mixture approach will be the only approach available to describe the inhomogeneous porous aluminum described in Sec. V.

\section{REFERENCE INHOMOGENEOUS POROUS MATERIAL}

The experimental method used to create an inhomogeneous porous material is depicted in Fig. 1 where a tapered cylinder of highly compressible melamine foam is forced inside a constant diameter tube (the acoustic measurements impedance tube). For the model, the porous cylinder is subdivided in a finite number of homogeneous and isotropic slices of thickness

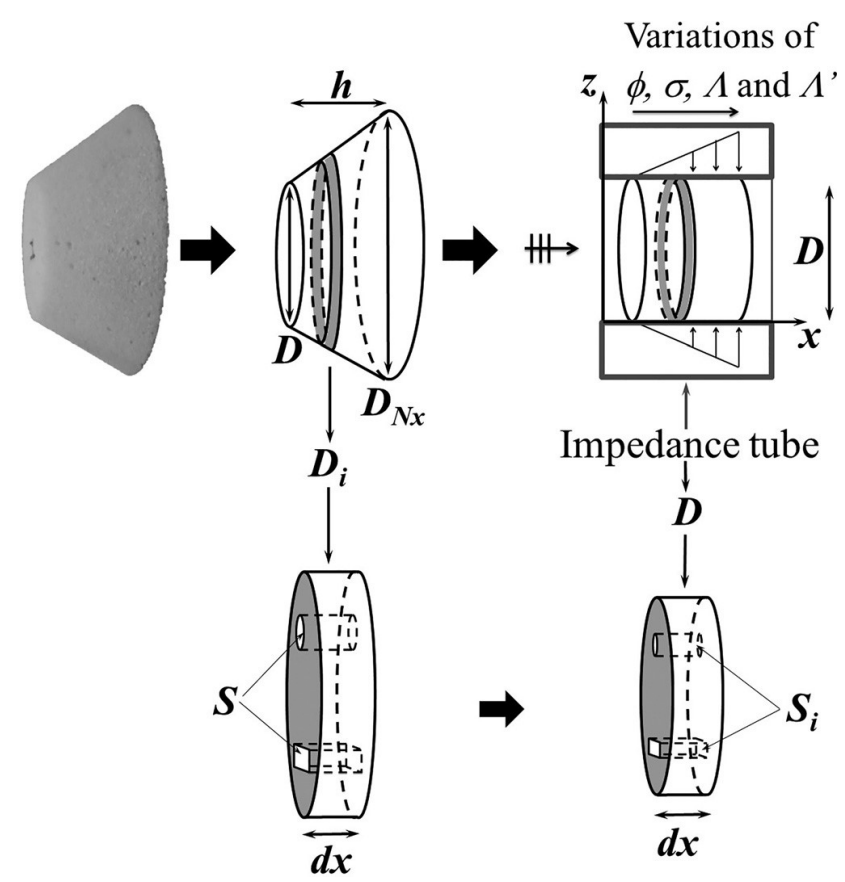

FIG. 1. Truncated conical melamine foam sample forced inside a rigid tube, here the measurement impedance tube (top). Discretization in thin slices of the radially compressed cylindrical foam sample (bottom).

$d x$ and the physical properties of the material are modified progressively from one face of the cylinder to the other as the static radial compression applied increases with depth. $D$ is the small diameter, which is also the impedance tube diameter, $D_{N x}$ the base diameter, and $x$ and $z$ the coordinate axis. The waves in the impedance tube are supposed to be plane waves, and the incidence is supposed to be normal to the sample.

In an idealized model, the layer thickness $h$ is constant before and after compression. However, it will be seen in the experimental part that this idealized assumption is not verified. This problem will be overcome by carrying out the experiments on two different samples cut out of the same material but with different initial thicknesses. More details are given in the comparison between the experimental results and the models in Sec. IV A.

The physical parameters of the melamine foam used to create a cone shape sample were determined using classical experimental methods or indirect methods. ${ }^{14-18}$ Their values are given in the first column of Table I in Sec. IV.

\section{MODEL FOR THE MICROSTRUCTURAL PARAMETERS OF THE REFERENCE INHOMOGENEOUS POROUS MATERIAL}

Castagnède et al. ${ }^{5}$ studied in the equivalent fluid approximation the effect on the physical parameters of applying a 1D uniaxial compression perpendicularly to the direction of a set of straight, parallel fibres of infinite length or a 2D compression radially to the direction of the fibres and they found expressions of the porosity, the tortuosity, the flow resistivity and the viscous and thermal characteristic lengths as functions of the parameters in the uncompressed state and of the radius $a$ of the fibres, 
In the present study where a conical shape cylinder of melamine foam undergoes radial compression increasing with depth (see Fig. 1, top), the inhomogeneous material can be modelled as a stacking of $N_{x}$ layers. It is found that the same expression as in Ref. 5 can be used for the porosity and the tortuosity,

$$
\begin{aligned}
& \phi_{i}=1-(1-\phi)\left(\frac{D_{i}}{D}\right)^{2} \\
& \alpha_{i}=1+\left(\alpha_{\infty}-1\right)\left(\frac{D_{i}}{D}\right)^{2},
\end{aligned}
$$

where $\phi_{i}$ is the of layer $i, \phi$ the open porosity before compression, $D$ the diameter of layer 1 and $D_{i}$ the diameter of layer $i$ before compression. The ratio $\left(D_{i} / D\right)$ is the compression rate (parameter $n$ in Ref. 5).

For the other parameters used in the TMM method (flow resistivity $\sigma_{i}$, viscous $\Lambda_{i}$ and $\Lambda_{i}^{\prime}$ thermal characteristic lengths), the expressions of Castagnède et al. cannot be used as the materials and the compression processes are different. However, a model for these parameters can be developed if a simplifying assumption is made. It is assumed that the pore microstructure geometry is maintained throughout the thickness of the sample and that only the pore sizes, not the geometries change with the compression. In this approach the solid, which is much less compressible than the fluid rearranges itself during a compression but is not submitted to variations of volumes. Although it looks drastic at first sight, it will be seen that this assumption provides a good theoretical prediction of the acoustic properties of the inhomogeneous material.

For a thin slice of material of diameter $D_{i}$ and thickness $d x$ [Fig. 1 (bottom)], the cumulative pore surface at the entry face of the layer before compression is denoted $S$ and that for the layer after compression is denoted $S_{i}$. The corresponding total pore volumes are obtained after integration along the thickness. The total volumes are given by, respectively,

$$
\begin{aligned}
& V=\int_{0}^{d x} S d x^{\prime}, \\
& V_{i}=\int_{0}^{d x} S_{i} d x^{\prime} .
\end{aligned}
$$

Under the previous assumption on the pore microgeometry, the cross sectional area of the pore is reduced by the same factor as the reduction in the cross sectional area of the cylindrical layers before and after compression. In addition, if the compression is considered constant along the $x$ axis inside a thin slice of thickness $d x, S$, and $S_{i}$ can be taken out of the integrals in Eqs. (3) and (4). The total pore volume $V$ or $V_{i}$ for one slice is therefore given by multiplying the cumulative cross section $S$ or $S_{i}$ by $d x$. The porosity of a slice before and after compression are given by dividing the volumes $V$ and $V_{i}$ by the total volumes of the cylinders, respectively,

$$
\phi=\frac{S d x}{d x \pi D_{i}^{2} / 4}=\frac{S}{\pi D_{i}^{2} / 4},
$$

$$
\phi_{i}=\frac{S_{i}}{\pi D^{2} / 4} .
$$

Since

$$
\frac{S_{i}}{S}=\left(\frac{\Lambda_{\mathrm{i}}^{\prime}}{\Lambda^{\prime}}\right)^{2}
$$

combining Eqs. (5), (6), and (7) provide the following ratio of porosities,

$$
\frac{\phi_{i}}{\phi}=\left(\frac{\Lambda_{\mathrm{i}}^{\prime}}{\Lambda^{\prime}}\right)^{2}\left(\frac{\mathrm{D}_{\mathrm{i}}}{\mathrm{D}}\right)^{2},
$$

and $\Lambda_{\mathrm{i}}^{\prime}$ can be expressed as

$$
\Lambda_{\mathrm{i}}^{\prime}=\left(\frac{\mathrm{D}}{\mathrm{D}_{\mathrm{i}}}\right) \sqrt{\frac{\phi_{i}}{\phi}} \Lambda^{\prime} .
$$

Similarly, the other geometrical parameter $\Lambda_{\mathrm{i}}$ is given by

$$
\Lambda_{\mathrm{i}}=\left(\frac{\mathrm{D}}{\mathrm{D}_{\mathrm{i}}}\right) \sqrt{\frac{\phi_{i}}{\phi}} \Lambda \text {. }
$$

Therefore, $\Lambda_{i}^{\prime}$ and $\Lambda_{i}$, can be expressed as functions of the porosity profile and of $\Lambda^{\prime}$ and $\Lambda$ by combining Eqs. (1), (9) and (1), (10),

$$
\begin{aligned}
& \Lambda_{\mathrm{i}}^{\prime}=\left[\left(\frac{1-\phi}{1-\phi_{i}}\right)\left(\frac{\phi_{i}}{\phi}\right)\right]^{1 / 2} \Lambda^{\prime}, \\
& \Lambda_{\mathrm{i}}=\left[\left(\frac{1-\phi}{1-\phi_{i}}\right)\left(\frac{\phi_{i}}{\phi}\right)\right]^{1 / 2} \Lambda .
\end{aligned}
$$

For the static airflow resistivity, the Carman-Kozeny law is used in which a shape factor $A$ is supposed constant for a homothetic transformation where the pore microgeometry is maintained radially. After Carman-Kozeny (see Bourbié et al., ${ }^{19}$ p. 35), the static permeability $\kappa$ is given by

$$
k=\frac{A}{\alpha_{\infty}} R_{H}^{2} \phi
$$

and the airflow resistivity is given by $\sigma=\eta / \kappa$, with $\eta$ the dynamic viscosity. In fact, $R_{H}$ the hydraulic radius corresponds to $\Lambda^{\prime}$ The parameters $A$ is a shape factor which is supposed constant under the assumption of constant microstructure geometry. It is then possible to obtain for a given slice $i$ a ratio $\sigma_{i} / \sigma$ for compressed and uncompressed slice of material by inserting $\kappa$ from Eq. (13) into the expression for $\sigma$ and considering the shape factor $A$ and the tortuosity constant. The flow resistivity $\sigma_{i}$ is given by

$$
\sigma_{i}=\left(\frac{1-\phi_{i}}{1-\phi}\right)\left(\frac{\phi}{\phi_{i}}\right)^{2}\left(\frac{\alpha_{\infty i}}{\alpha_{\infty}}\right) \sigma .
$$

The tortuosity ratio $\alpha_{\infty i} / \alpha_{\infty}$ can be considered to be equal to 1 in this study. In fact, using Eq. (2), the maximum value 
found for this ratio during a compression is 1.03. Using this model for the physical properties of the compressed material, the necessary parameters can all be expressed as functions of $\phi_{i}$ and only a porosity profile (or volume proportion of materials $A$ and $B$ ) is required to describe the macroscopic inhomogeneity in the material.

\section{COMPRESSED TAPERED POROUS CYLINDER}

\section{A. Comparison between the TMM and experimental results}

The acoustic indicators of multi-layered systems can be described with the help of transfer matrices in the wellknown TMM approach in the rigid or limp frame approximation (see Allard and Atalla, ${ }^{9}$ Chap. 11). The TMM method can be used to describe inhomogeneous materials by considering a great number $N_{x}$ of thin layers of thickness $d x$. The material properties are involved in the elements of the transfer matrices through the use of the complex wave number $k_{i}$ and characteristic impedance $Z_{c i}$ for each layer $i$. These can be calculated with the help of the model [Eqs. (1), (2), (11), (12), and (14)].

A tapered cylinder of smaller diameter $D=29.8 \pm 0.3 \mathrm{~mm}$ and base diameter $D_{N x}=41.3 \pm 0.2 \mathrm{~mm}$ forced inside a $29 \mathrm{~mm}$ impedance tube is studied experimentally and theoretically [Eqs. (1), (2), (11), (12), and (14) and TMM method]. The results on the transmission loss (TL) are presented in Fig. 2. The thickness of the "compressed" sample was measured inside the tube and was $25.6 \mathrm{~mm}$. A second sample of same thickness and of diameter $29 \mathrm{~mm}$ was then cut out of the same material (melamine). This second sample acted as a "not compressed" reference sample when introduced in the tube. This method allows the comparison between theoretical and experimental results on a compressed or uncompressed material without having to apply a thickness correction.

The simulations were done for $N_{x}=100$ stacked layers. The minimum discretization number of layers $N_{x}$ is determined from preliminary convergence tests. In Table I, the parameters of the TMM method have been measured in the case of the uncompressed sample and determined from

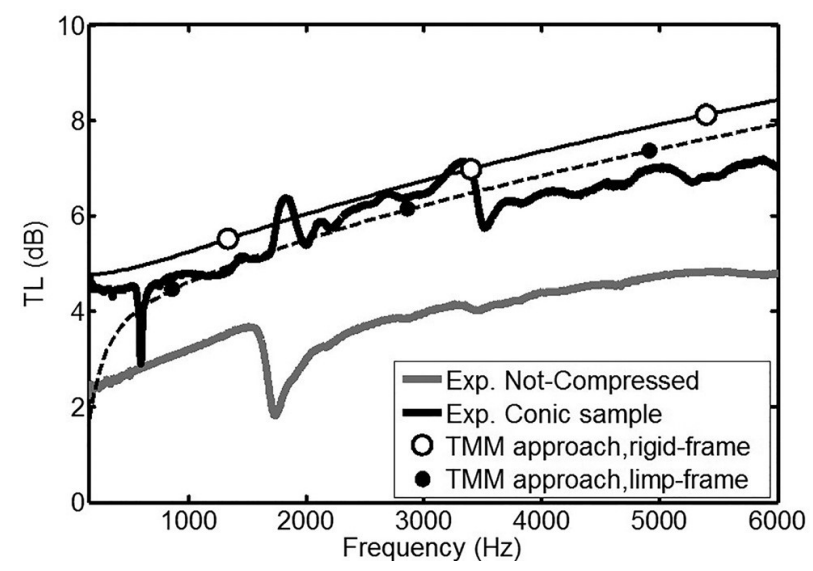

FIG. 2. Transmission loss measurement and prediction by the classical TMM approach for a compressed tapered cylinder or highly porous melamine foam.
TABLE I. Parameters in the TMM method for a not compressed melamine sample and for the first and the last equivalent layers of the compressed truncated conical sample.

\begin{tabular}{lccc}
\hline \hline & Not compressed & Layer 1 & Layer $N_{x}$ \\
\hline$\phi$ & $0.99 \pm 0.01$ & $0.98 \pm 0.01$ & $0.96 \pm 0.04$ \\
$\sigma\left(\mathrm{Pa} \mathrm{s} / \mathrm{m}^{2}\right)$ & $11987 \pm 431$ & $12679 \pm 582$ & $34855 \pm 1468$ \\
$\alpha_{\infty}$ & $1.02 \pm 0.01$ & $1.02 \pm 0.01$ & $1.05 \pm 0.03$ \\
$\Lambda(\mu \mathrm{m})$ & $80 \pm 9$ & $78 \pm 9$ & $48 \pm 5$ \\
$\Lambda^{\prime}(\mu \mathrm{m})$ & $138 \pm 34$ & $134 \pm 33$ & $82 \pm 20$ \\
$\rho\left(\mathrm{kg} / \mathrm{m}^{3}\right)$ & $8 \pm 3$ & $9 \pm 6$ & $21 \pm 15$ \\
\hline \hline
\end{tabular}

the model proposed in Sec. III in the case of compressed material. For the compressed material, the errors were deduced from the experimental errors in the uncompressed case.

The experimental results are well predicted with the multilayer decomposition in the TMM approach. The effect of radial compression on the conical sample was predicted and confirmed experimentally. The resonance due to the solid frame vibration is shifted to higher frequencies as expected. This effect was studied by Wang et al. ${ }^{6}$ for fibrous materials. The rigid and limp frame behaviors are also predicted, and the experimental curve seems to confirm these behaviors at low and high frequencies, respectively.,20

\section{B. Comparison between the experimental results and the mixture approach}

The present study is focused on a new approach for the study of macroscopically inhomogeneous materials based on a mixture approach for the calculation of the acoustic indicators of elements placed in parallel (or patch) configuration. The inhomogeneous material is viewed as a mixture of two materials and represented in Fig. 3. As the depth increases, one material (in white) is progressively replaced by another (in black). In this approach, in order to create a volume profile, discretization along the $z$ axis as well as along the $x$ axis must be introduced.

The first order homogenization procedure ${ }^{10,11}$ provides the effective density $\rho_{H}$ and effective bulk modulus $K_{H}$ of each layer $i$, respectively, from the harmonic average of the

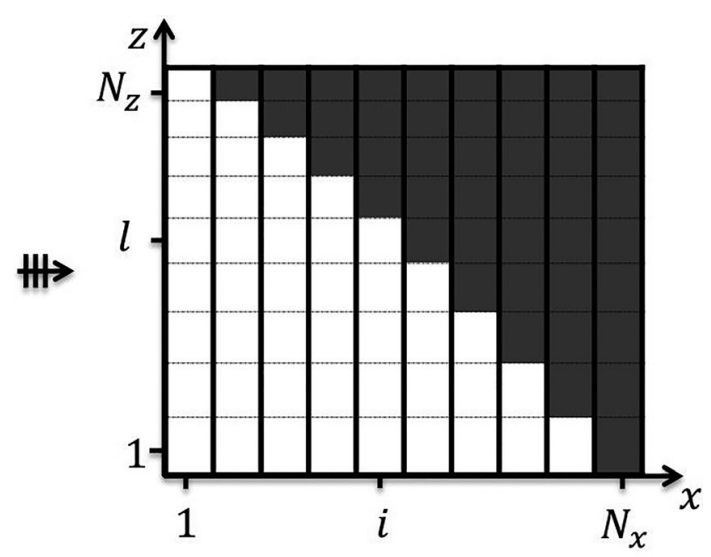

FIG. 3. Stacking in which the white square column represent the first layer of equivalent material of diameter $D$ and the dark square column represent the last (most compressed) layer of diameter $D_{N x}$. 
densities $\left(\rho_{1}\right.$ and $\left.\rho_{2}\right)$ of the two porous materials and from their bulk moduli $\left(K_{1}\right.$ and $\left.K_{2}\right)$,

$$
\rho_{H}=\left(\frac{r_{1}}{\rho_{1}}+\frac{r_{2}}{\rho_{2}}\right)^{-1} \text { and } K_{H}=\left(\frac{r_{1}}{K_{1}}+\frac{r_{2}}{K_{2}}\right)^{-1} \text {, }
$$

where $r_{1}$ and $r_{2}$ represent the surface area ratios occupied by material 1 and 2, respectively, with respect to the total surface area. The characteristic impedance (or admittance) of layer $i$ can then be determined from its density and bulk modulus. In our model where the material is cut in $N_{x}$ slices, the thickness is very small and corresponds to $d x$ in Fig. 1. The characteristic impedance of layer $i$ is then given by

$$
Z_{i}=\sqrt{\left(\rho_{H}\right)_{i}\left(K_{H}\right)_{i}} .
$$

The effective wavenumber $\left(k_{M R M}\right)_{i}$ for layer $i$ is also deduced from $\rho_{H}$ and $K_{H}$. Equations (15) are then reinjected in the classical transfer matrices through $Z_{i}$. This allows the determination of the acoustic indicators. In the mixture approach, as illustrated in Fig. 3, a layer $i$ only involves the parameters of the first layer (material " $A$, , $i=1$ ), of the last layer (material " $B$," $i=N_{x}$ ) and their respective proportion illustrated by the discretization along the $z$ axis. The variation in the macroscopic properties of a compressed truncated conical sample is considered as due to a variation of the volume ratio of a mixture of a not compressed material (corresponding to layer 1) and of the most compressed material (corresponding to layer $N_{x}$ ). This volume ratio varies from $0 \%$ (material " $A$ " in white, not compressed) to $100 \%$ (material " $B$ " in black, most compressed) and is directly related to the local porosity [Eq. (1)]. In this description, only two materials are staked in a patch configuration in a layer $i$. The surface ratio and the porosity of both materials determine the local porosity,

$$
\phi_{i}=r_{i, \mathrm{mat}_{1}} \phi+r_{i, \mathrm{mat}_{N x}} \phi_{N x},
$$

and a mixing law can be defined from the porosity profile $\phi_{i}$,

$$
r_{i}=\frac{\phi_{N x}-\phi_{i}}{\phi_{N x}-\phi}
$$

The surface ratios of both materials obeys the following relationship:

$$
\left(r_{\text {mat }_{1}}\right)_{i}+\left(r_{\text {mat }_{N x}}\right)_{i}=1 .
$$

The surface ratios correspond to volume ratios for slices of same thickness. The originality of this approach is that only the properties (physical parameters) of the first and last layers are necessary, the inhomogeneity being accounted for through a variation of volume proportion of the first and last layers. However, this approach assumes that the first and last layers have the same microstructural geometry. For validation, the transmission loss determined by the mixture approach [Eqs. (15) and (16)] is plotted in Fig. 4 in Sec. IV C.

\section{Experimental validation of the mixture approach}

For the same reference sample studied earlier in Sec. IV A, the transmission loss calculated from the mixture approach and the mixture approach is compared to the experimental results in Fig. 4. The experimental results are the same as in Fig. 2. The number of layers along $x$ is also taken to be $N_{x}=100$. The number of elements along the $z$ axis is $N_{z}=99$ (see Fig. 3). A preliminary convergence analysis has also been performed to determine these numbers.

A good agreement between experimental and theoretical results is also found. However, it is clear that the mixture approach (Fig. 4) does not provide identical results as the theoretical model of inhomogeneous materials (Fig. 3) obtained from modelling the pore compression. The discrepancy between the two approaches can be attributed to the additional assumptions on the pore microgeometry made in the mixture approach. The theoretical results of Fig. 3 can be considered as more rigorous. However, the advantage of the mixture approach is its capacity to predict the properties of a graded material with only the physical parameters of the two external layers (first and last layers) and only a "mixture profile" on the porosity as unknowns, while for the full inhomogeneous model, the parameters of all layers have to be known. This represents a key advantage in Sec. V where only the mixture approach can be used in the study of an inhomogeneous porous aluminum foam obtained from a process where two particle sizes salt grains are mixed.

\section{ACOUSTICAL PROPERTIES OF AN INHOMOGENEOUS ALUMINUM FOAM}

\section{A. Aluminum foam fabrication}

The porous aluminum foams can be obtained after melted aluminum has invaded the pore space of a sample of salt grains at negative pressure and high temperature (Fig. 5, Gong et al. ${ }^{21}$ ). After cooling and solidification of the metal, a porous aluminum can be obtained by dissolving out the salt grains by water washing at ambient temperature. The distribution in the pore size can be obtained by controlling the initial salt grain particle size distribution by successive sieving of the salt grains. After the salt grains have been dissolved, the sample is dried and the space formerly occupied

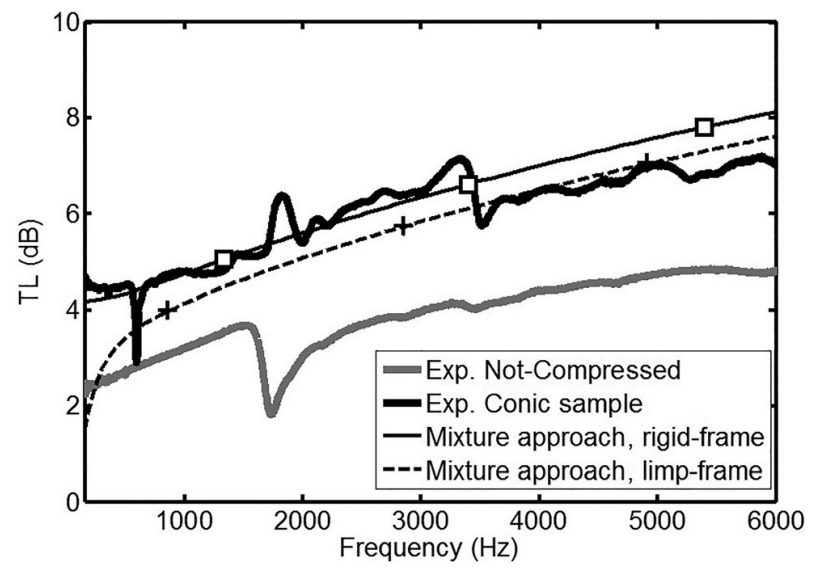

FIG. 4. Transmission loss measurement and simulation by the mixture approach for the tapered cylinder and uncompressed homogenous melamine. 


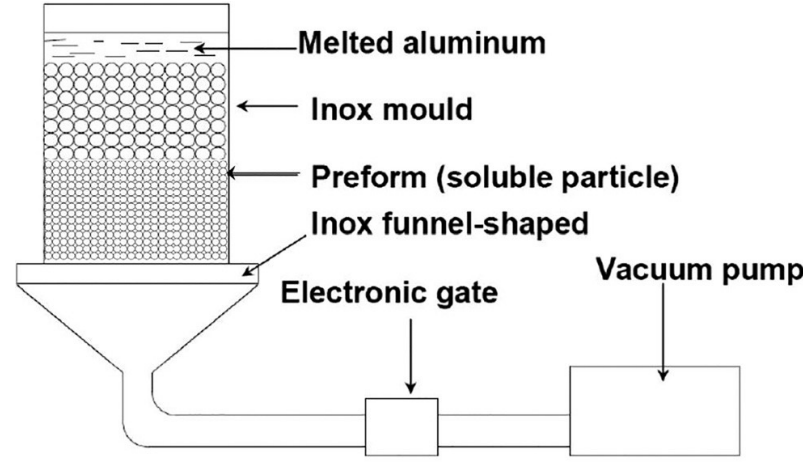

FIG. 5. Principle of the fabrication of an inhomogeneous aluminum foam [Redrawn after Gong et al. (Ref. 21)].

by the grains are replaced by air so that the porous aluminum is created.

An inhomogeneous sample of aluminum foam was built using two sizes of salt grains. The analysis of microscope photographs of a cross-sections of the sample reveals that this metallic foam is composed of three layers: two homogeneous layers of different materials and an inhomogeneous interface layer (localized between the two homogeneous layers). A progressive mix of the cells of the two homogeneous layers occurs in the intermediate interface layer. Based on this observation, the inhomogeneous aluminium foam is identified as a multi-layered material composed of three different materials stacked in series.

\section{B. Mixture approach for the inhomogeneous porous aluminum}

The macroscopic physical parameters of the two homogeneous layers can be determined separately. Separate samples of each homogeneous layers were fabricated prior to this study and their parameters were determined. For the inhomogeneous interface layer, the "mixture" of two materials is performed by varying volume proportions from one material to the other. The created material is identified as a porous material with a porosity profile along the thickness of the sample (see Fig. 3).

Implementing the classical TMM approach is not possible as the physical properties of the $N_{x}$ discretized layers cannot be accessed. The mixture approach however can be more easily applied as the volume proportion profile in the interface layer can be evaluated. This leads to the determination of the transfer matrix of the intermediate layer. The global transfer matrix of the global inhomogeneous metallic foam is then determined by the product of transfer matrices of the three layers (see Fig. 6). The metallic porous frame is considered to be rigid framed. The inhomogeneous metallic foam presented in this study has been made with salt grains of two classes of sizes (the grain average diameters are between 0.9 and $1.1 \mathrm{~mm}$ for class $A$ and between 1.4 and $1.8 \mathrm{~mm}$ for class $B$ ) [see Fig. 6(a)]. The two homogenous layers correspond to the two metallic foams MF1 and MF2 for which the physical parameters are given by using the classical method (Refs. 14-18, measurements and indirect

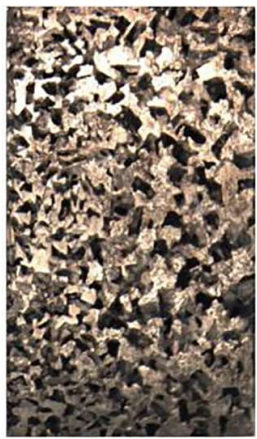

a)

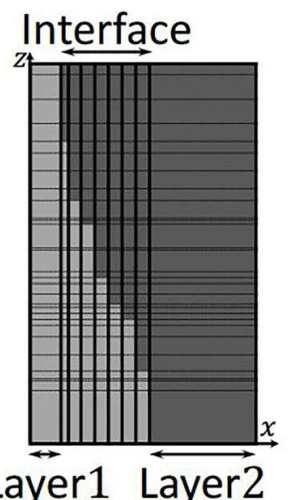

)

b)
FIG. 6. (Color online) (a) Cut of the metallic foam. (b) Representation in mixture approach by a three-layered system with the help of an optimization procedure.

method are performed on separate measurements on samples class $A$ and $B$ ). The results are given in Table II.

The global sample thickness is $h=14.8 \mathrm{~mm}$ in both cases. With the help of a microscope analysis [see Fig. 6(a)] and an optimization approach (algorithm of simulated annealing, Ref. 22), the thickness of each specific layer is defined. The thicknesses of the first homogenous layer, of the inhomogeneous interface layer and of the second homogenous layer are, respectively, 4.0, 2.5, and $8.3 \mathrm{~mm}$.

The interface parameters for the implementation of the mixture approach have been chosen to be $N_{x}=9$ layers and $N_{z}=30$ columns. $N_{x}$ and $N_{z}$ result from preliminary convergence analysis and the simulated annealing optimization algorithm (see Ref. 22). This optimization techniques is based from an analogy with the annealing process behaviour and consists basically in the iterative improvement of a cost function. ${ }^{22}$ This function represents a quantitative measure of the "quality" of a complex system and was used in statistical mechanics.

In the implementation of the simulated annealing optimization, the total thickness of the inhomogeneous material is necessary as well as the thicknesses and physical parameters of the first and last homogeneous layers, the number of sublayers in the interface layer and the total number of elements stacked in along the $z$ axis. A frequency array and the TL curve to be fitted are also required. The simulated annealing also need specific parameters including the initial temperature in the simulation of annealing, a cooling function, a maximum number of iteration and a number of iterations per temperature step. Constraints on the limit values are then

TABLE II. Physical parameters of homogenous aluminum foam sample.

\begin{tabular}{lcc}
\hline \hline & MF1 (class A) & MF2 (class B) \\
\hline$H(\mathrm{~mm})$ & 14.9 & 14.4 \\
$\Phi$ & $0.64 \pm 0.01$ & $0.66 \pm 0.01$ \\
$\sigma\left(\mathrm{Pa} \mathrm{s} / \mathrm{m}^{2}\right)$ & $4316 \pm 212$ & $3190 \pm 212$ \\
$\alpha_{\infty}$ & $1.6 \pm 0.2$ & $1.7 \pm 0.2$ \\
$\Lambda(\mu \mathrm{m})$ & $170 \pm 13$ & $223 \pm 9$ \\
$\Lambda^{\prime}(\mu \mathrm{m})$ & $196 \pm 29$ & $544 \pm 24$ \\
\hline \hline
\end{tabular}




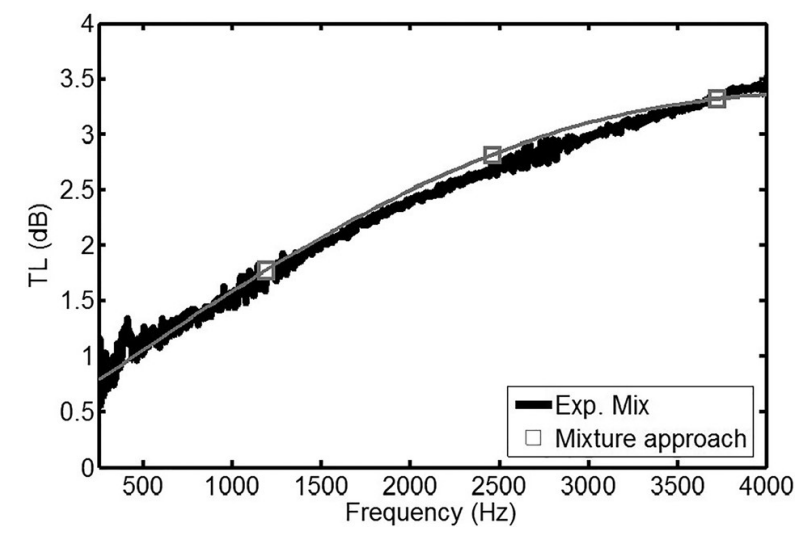

FIG. 7. Transmission loss comparison between measurements on a mixture of MF1 and MF2 size cells sample and the mixture approach.

introduced and an initial guess by a normally distributed random number is used. The cost function to be minimized is given by

$$
f=\sum_{k}\left|\mathrm{TL}_{\exp }\left(f_{k}\right) \quad \mathrm{TL}_{\text {model }}\left(f_{k}\right)\right|^{2},
$$

where $\mathrm{TL}_{\text {exp }}\left(f_{k}\right)$ and $\mathrm{TL}_{\text {model }}\left(f_{k}\right)$ correspond to the experimental value of the transmission loss and the transmission loss given by the model (mixture approach) at the frequency $f_{k}$.

The minimization provides the topology of the volume proportion profile inasmuch as it says whether the $i$ th and $l$ th rectangle in Fig. 3 should be white (material A) or black (material B). This annealing optimization algorithm was applied to the aluminium foam and provided the interface profile representing the "mixture." Figure 6 shows the correspondence between the observed by visual microscopy [Fig. 6(a)] and the interface profile obtained method and by the optimization method [Fig. 6(b)].

The experimental results and the prediction of the transmission loss based on the mixture approach and using the physical parameters of the first and last layers are compared in Fig. 7. It is noticed that the present modelling compares very well with the experimental results and seems well adapted to describe the acoustic properties of bi-layer metallic foams with a mixed-cells interface provided the property gradient is not too strong.

\section{CONCLUSIONS}

The transmission loss of macroscopically inhomogeneous porous layers saturated by air in the rigid or limp frame approximation were studied through the use of a radial compression varying with depth in a cylindrical sample. A reference material obtained by forcing a tapered cylinder of highly compressible porous material inside a rigid constant cross section tube was first built and studied.

Assuming that the pore geometry is maintained throughout the thickness of the material during a radial compression, it was possible to model the classical transfer matrix for the reference material. Experimental results on the transmission loss were obtained and validated the assumption on the pore geometry.
In view of studying an inhomogeneous aluminium foam, a new approach based on the "mixing" of two known materials was proposed. In this approach, the two materials have volume proportions varying with depth and are placed in a patchwork configuration. With the help of the first order homogenization procedure, it was possible to model the acoustic indicators. For the reference material, this approach could be compared to the experimental results and to the results of the classical TMM for validation.

The mixture approach could be then used to model an inhomogeneous aluminium foam sample for which only the physical properties of the external faces were known. The volume proportion profile was recovered from a simulated annealing optimization process. The comparison with experimental results confirmed the validity of the mixture approach.

\section{ACKNOWLEDGMENTS}

This work was supported by the Ministère de l'Enseignement Supérieur et de la Recherche (France) and by the National Sciences and Engineering Research Council of Canada.

\section{APPENDIX}

Comparison between the classical admittance sum method (ASM) and the first order homogenization procedure $(\mathrm{H})$.

It was mentioned that the mixing approach used in this article needed a mixing rule. It is shown that under certain circumstances, another mixing rule, namely, the classical ASM can provide results very similar to the first order homogenization procedure. The ASM method can be very convenient especially when used in combination with the parallel transfer matrix method. ${ }^{12}$ It is proposed to compare the global transfer matrix calculated by the first order homogenization $^{10,11}$ and the global transfer matrix calculated by the ASM, which is embedded in the P-TMM for a thin patchwork or for low frequencies. Consider a patchwork which is composed by two materials (or elements) of same thickness $\delta h$ :

Material 1: with density and bulk modulus respectively $\rho_{1}, K_{1}$ occupying a surface ratio $r_{1}$ of the patchwork.

Material 2: with density and bulk modulus, respectively, $\rho_{2}, K_{2}$ occupying a surface ratio $r_{2}=1-r_{1}$ of the patchwork.

The homogenization procedure ${ }^{10,11}$ gives the global macroscopic parameters $\rho_{H}$ and $K_{H}$ as

$$
\begin{aligned}
\rho_{H} & =\left(\frac{r_{1}}{\rho_{1}}+\frac{r_{2}}{\rho_{2}}\right)^{-1} \\
K_{H} & =\left(\frac{r_{1}}{K_{1}}+\frac{r_{2}}{K_{2}}\right)^{-1} .
\end{aligned}
$$

The global transfer matrix of a symmetric slice of the patchwork is given by

$$
\left[T_{H}\right]=\left[\begin{array}{cc}
\cos \left(k_{H} \delta h\right) & j Z_{H} \sin \left(k_{H} \delta h\right) \\
\frac{j}{Z_{H}} \sin \left(k_{H} \delta h\right) & \cos \left(k_{H} \delta h\right)
\end{array}\right]
$$


with the wavenumber and the characteristic impedance of the homogenized equivalent fluid given by $k_{H}=\omega \sqrt{\rho_{H} / K_{H}}$ and $Z_{H}=\sqrt{\rho_{H} K_{H}}$.

For a small thickness or low frequencies $\left[\left(k_{H}\right)_{i} \delta h \ll 1\right]$, the global transfer matrix can be approximated as follows (first order Taylor's expansion):

$$
\left[T_{H}\right] \cong\left[\begin{array}{cc}
1 & j \omega \rho_{H} \delta h \\
\frac{j \omega \delta h}{K_{H}} & 1
\end{array}\right]
$$

and using the expressions of $\rho_{H}$ and $k_{H}$, the transfer matrix of the slice of patchwork sample is given by

$$
\left[T_{H}\right] \cong\left[\begin{array}{cc}
1 & j \omega \delta h\left(\frac{r_{1}}{\rho_{1}}+\frac{r_{2}}{\rho_{2}}\right)^{-1} \\
j \omega \delta h\left(\frac{r_{1}}{K_{1}}+\frac{r_{2}}{K_{2}}\right) & 1
\end{array}\right]
$$

In the admittance sum embedded in the parallel transfer matrix method, since the elements are in parallel, it is more convenient to work with global admittances $[Y]$ given as a function of the admittance matrix of the lateral sub-layers $\left[Y_{i}\right]$,

$$
[Y]=\sum_{i}^{N x} r_{i}\left[Y_{i}\right]
$$

A parameter $r_{i}$ being defined as the surface ratio of a given element $i$ and considering $N_{z}$ elements stacked along the $z$ axis, the total admittance $[Y]$ is given by

$$
\left[Y_{i}\right]=\frac{1}{t_{i, 12}}\left[\begin{array}{ccc}
t_{i, 22} & t_{i, 21} t_{i, 12} & t_{i, 22} t_{i, 11} \\
1 & & t_{i, 11}
\end{array}\right]
$$

where $t_{i, 11}=t_{i, 22}=\cos \left(k_{i} l\right), \quad t_{i, 12}=j Z_{C i} \sin \left(k_{i} l\right), \quad t_{i, 21}=j(1 /$ $\left.Z_{C i}\right) \sin \left(k_{i} l\right)$ are the elements of the matrix $\left[Y_{i}\right]$ (see Ref. 11) in which the subscript $i$ corresponds to layer $i, k_{i}$, and $Z_{C i}$ are, respectively, the equivalent wavenumber and the characteristic impedance in material $i$.

In the P-TMM approach, the global transfer matrix of the $i$ th patch ( $i$ is equal to 1 or 2 here) is given by

$$
\left[T_{P T M M}\right]=\frac{-1}{\sum r_{i} y_{i, 21}}\left(\begin{array}{cc}
\sum r_{i} y_{i, 22} & -1 \\
\sum r_{i} y_{i, 22} \sum r_{i} y_{i, 11}-\sum r_{i} y_{i, 12} \sum r_{i} y_{i, 21} & \sum r_{i} y_{i, 11}
\end{array}\right)
$$

$r_{i}$ corresponds to the surface ratio of patch $i$ and the $y_{i, n m}$, terms are the coefficients of the admittance matrix. The admittance matrix is given by [see Eq. (A6)]

$$
\begin{aligned}
{\left[Y_{i}\right] } & =\left[\begin{array}{ll}
y_{i, 11} & y_{i, 12} \\
y_{i, 21} & y_{i, 22}
\end{array}\right] \\
& =\left[\begin{array}{cc}
\frac{\cos \left(k_{i} \delta h\right)}{j Z_{C i} \sin \left(k_{i} \delta h\right)} & \frac{1}{j Z_{C i} \sin \left(k_{i} \delta h\right)} \\
\frac{1}{j Z_{C i} \sin \left(k_{i} \delta h\right)} & \frac{\cos \left(k_{H} \delta h\right)}{j Z_{C i} \sin \left(k_{i} \delta h\right)}
\end{array}\right] .
\end{aligned}
$$

For a patchwork constituted of materials 1 and 2 and in the thin material or low frequency approximation $\left[\left(k_{M R M}\right)_{i} \delta h\right.$ $\ll 1$ ], the global transfer matrix takes a simple form,

$$
\left[T_{P T M M}\right] \cong\left[\begin{array}{cc}
1 & j \omega \delta h\left(\frac{r_{1}}{\rho_{1}}+\frac{r_{2}}{\rho_{2}}\right)^{-1} \\
j \omega \delta h\left(\frac{r_{1}}{K_{1}}+\frac{r_{2}}{K_{2}}\right) & 1
\end{array}\right],
$$

which is the same as Eq. (A4). It can be concluded that for a patchwork with a thin material or at low frequencies, the P-TMM and the first order homogenization procedure give the same global transfer matrix for the patchwork.

For the compressed inhomogeneous melamine foam of Fig. 1, the transmission loss provided by the use of homogenization procedure or P-TMM are plotted against frequency in Fig. 8 in the rigid frame approximation. The error is small and the two curves cannot be distinguished. The maximum error was less than $3 \%$ and corresponded to a surface ratio close to 0.5 , i.e., around the middle of the compressed tapered cylinder of the melamine constituted of the patchwork of materials $\mathrm{A}$ and B (see Fig. 3). The results of Fig. 8 correspond to the plain curve of Fig. 4. The comparison

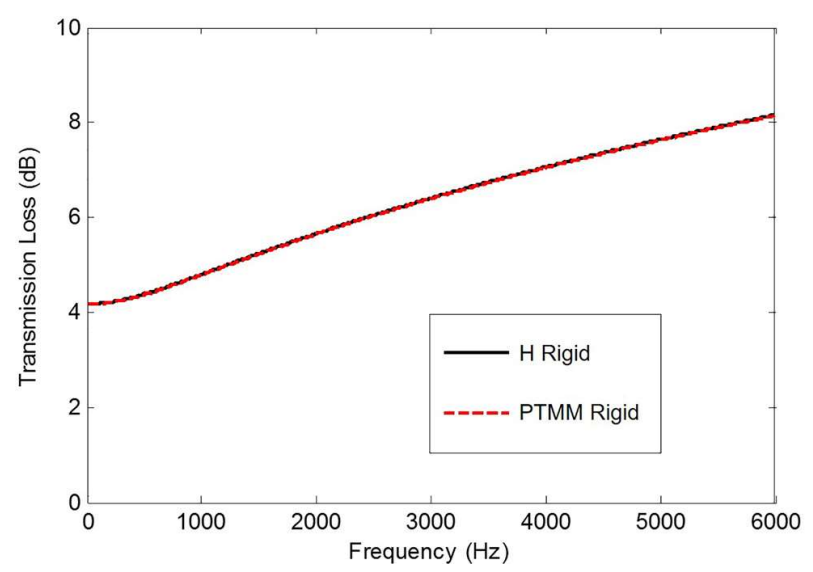

FIG. 8. (Color online) Comparison between the first order homogenization procedure $(\mathrm{H})$ and ASM (or PTMM) for the compressed cone shape melamine foam (in the rigid frame approximation case). 
between first order homogenization procedure and PTMM using the limp frame approximation also provided a very good match.

It should be noted that this result could be expected as the compressed melamine has been subdivided in a great number of slices of thickness $d x$ so that the first order homogenization procedure or ASM were implemented for each slice under the thin slice material approximation, even though the ASM does not account for possible interactions between the different patches. The method works well because the slices are very thin so that it does not requires a clear definite separation of the fluxes as these cannot build up through the lateral dimensions before encountering the next slice. This approximation can be regarded as similar in principle to the use of rectangles in a numerical integration process instead of trapezoidal shapes. The accuracy can be increased at will by reducing the slice thickness. In this approximation, the two methods have the same asymptotic behavior.

${ }^{1}$ L. De Ryck, J. P. Groby, P. Leclaire, W. Lauriks, A. Wirgin, Z. E. A. Fellah, and C. Depollier, "Acoustic wave propagation in a macroscopically inhomogeneous porous medium saturated by a fluid," Appl. Phys. Lett. 90, 181901 (2007).

${ }^{2}$ L. De Ryck, W. Lauriks, Z. E. A. Fellah, A. Wirgin, J. P. Groby, P. Leclaire, and C. Depollier, "Acoustic wave propagation and internal fields in rigid frame macroscopically inhomogeneous porous media," J. Appl. Phys. 102, 024910 (2007).

${ }^{3}$ G. Gautier, L. Kelders, J. P. Groby, O. Dazel, L. De Ryck, and P. Leclaire, "Propagation of acoustic waves in a one-dimensional macroscopically inhomogeneous poroelastic material," J. Acoust. Soc. Am. 130, 1390-1398 (2011).

${ }^{4}$ A. Geslain, J. P. Groby, O. Dazel, S. Mahasaranon, K. V. Horoshenkov, and A. Khan, "An application of the Peano series expansion to predict sound propagation in materials with continuous pore stratification," J. Acoust. Soc. Am. 132, 208-215 (2012).

${ }^{5}$ B. Castagnède, A. Aknine, B. Brouard, and V. Tarnow, "Effects of compression on the sound absorption of fibrous materials," Appl. Acoust. 61, 173-182 (2000).

${ }^{6}$ C. N. Wang, Y. M. Kuo, and S. K. Chen, "Effects of compression on the sound absorption of porous materials with elastic frame," Appl. Acoust. 69, 31-39 (2008)
${ }^{7}$ B. Campolina, N. Dauchez, N. Atalla, and O. Doutres, "Effect of porous material compression on the sound transmission of a covered single leaf panel," Appl. Acoust. 73, 791-797 (2012).

${ }^{8}$ A. Geslain, O. Dazel, J.-P. Groby, S. Sahraoui, and W. Lauriks, "Influence of static compression on mechanical parameters of acoustic foams," J. Acoust. Soc. Am. 130, 818-825 (2011).

${ }^{9}$ J. F. Allard and N. Atalla, Propagation of Sound in Porous Media: Modelling Sound Absorbing Materials, 2nd ed. (John Wiley and Sons, New York, 2009), Chap. 11

${ }^{10}$ A. Bensoussan, J. L. Lions, and G. Papanicolaou, Asymptotic Analysis for Periodic Structures, Studies in Mathematics and Its Applications (NorthHolland, Amsterdam, 1978), Vol. 5, 700 pp.

${ }^{11} \mathrm{X}$. Olny and C. Boutin, "Acoustic wave propagation in double porosity media,” J. Acoust. Soc. Am. 114, 73-89 (2003).

${ }^{12}$ K. Verdière, R. Panneton, S. Elkoun, T. Dupont, and P. Leclaire, "Transfer matrix method applied to the parallel assembly of sound absorbing materials," J. Acoust. Soc. Am. 134, 4648-4658 (2013).

${ }^{13}$ K. Verdière, R. Panneton, S. Elkoun, T. Dupont, and P. Leclaire, "Comparison between parallel transfer matrix method and admittance sum method," J. Acoust. Soc. Am. 136, EL90-EL95 (2014).

${ }^{14} Y$. Salissou and R. Panneton, "Pressure/mass method to measure open porosity of porous solids," J. Appl. Phys. 101, 124913 (2007).

${ }^{15}$ P. Leclaire, L. Kelders, W. Lauriks, M. Melon, N. Brown, and B. Castagnède, "Determination of the viscous and thermal characteristic lengths of plastic foams by ultrasonic measurements in helium and air," J. Appl. Phys. 80, 2009-2012 (1996).

${ }^{16} \mathrm{R}$. Panneton and X. Olny, "Acoustical determination of the parameters governing viscous dissipation in porous media," J. Acoust. Soc. Am. 119, 2027-2040 (2006)

${ }^{17} \mathrm{X}$. Olny and R. Panneton, "Acoustical determination of the parameters governing thermal dissipation in porous media," J. Acoust. Soc. Am. 123, 814-824 (2008).

${ }^{18}$ ISO9053: Acoustics-Materials for Acoustical ApplicationsDetermination of Airflow Resistance (International Organization for Standardization, Geneva, Switzerland, 1991).

${ }^{19}$ T. Bourbié, O. Coussy, and B. Zinszner, Acoustique des Milieux Poreux (Acoustics of Porous Media) (Editions Technip, Paris, 1986)

${ }^{20} \mathrm{R}$. Panneton, "Comments on the limp frame equivalent fluid model for porous media,” J. Acoust. Soc. Am. 122, EL217-221 (2007)

${ }^{21}$ X. L. Gong, Y. Liu, S. Y. He, and J. Lu, "Manufacturing and low-velocity impact response of a new composite material: Metal porous polymer composite (mppc)," J. Mater. Sci. Technol. 20, 65-68 (2004).

${ }^{22}$ S. Kirkpatrick, C. D. Gerlatt, and M. P. Vecchi, "Optimization by simulated annealing," Science 220, 671-680 (1983) 